\title{
PROPERTY CONTROL METHODS OF DIAMOND-LIKE SILICON-CARBON FILMS FOR MICRO- AND NANOELECTRONICS
}

\author{
A.D. Barinov ${ }^{(a)}$, A.I. Popov ${ }^{(b)}$, A.A. Makarov $^{(c)}$ \\ (a),(b),(c) National Research University “Moscow Power Engineering Institute”, Moscow, Russia \\ (a),(b) Institute of Nanotechnology of Microelectronics of the Russian Academy of Science, Moscow, Russia

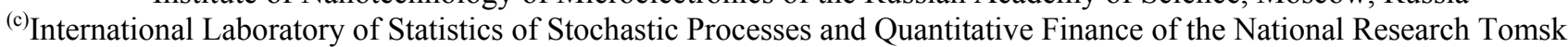 \\ State University, Tomsk, Russia \\ (a) $\underline{\text { barinovad@mpei.ru, }}{ }^{\text {(b) } 2009 \text { popovai@gmail.com, }}{ }^{(\mathrm{c})}$ o_v_ch@mail.ru
}

\begin{abstract}
Possible methods for controlling the properties of amorphous diamond-like silicon-carbon films are considered: physical or structural modification, chemical modification, and physical-chemical modification. It is shown that the method of physical modification allows controlling in a wide range the properties of diamondlike silicon-carbon films (electrophysical, mechanical properties and surface morphology) without changing the chemical composition of the material. Chemical modification was carried out by introducing transition metal into diamond-like silicon-carbon films. The dependences of the phase composition, electrophysical and mechanical properties on the content and type of metal are analyzed. The method of physical-chemical modification is considered, when the introduced impurity changes not only the chemical composition, but also the structure of the material.
\end{abstract}

Keywords: silicon-carbon films, diamond-like structure, modification of properties, phase composition, physical, chemical and physical-chemical modification, transition metals

\section{INTRODUCTION}

Diamond like amorphous carbon films consist of structural network in which carbon atoms mainly have $s p^{3}$ hybridization of electron orbits. These films are widely used in micro- and nanoelectronics, optoelectronics, microelectromechanical systems, aerospace engineering due to their high electrical resistivity, good optical transparency high hardness and low friction (Robertson 2002). At the same time there are some limitations in the application of diamond like amorphous carbon films. These relate to the limited adhesion due to a high intrinsic compressive stress, low thermal stability and material graphitization at high temperatures. In addition, as for all amorphous semiconductors, there are certain difficulties in controlling the properties of the material, due to their weak sensitivity to impurities.

Diamond-like silicon-carbon films are materials that retain the advantages of the amorphous diamond-like carbon films, but are largely free from many of its drawbacks (Mangolinia, Krick et al. 2018). These materials consist of hydrogenated amorphous carbon diamond like network $(a-\mathrm{C}: \mathrm{H})$ and an amorphous silica network ( $a$-Si:O) that mutually stabilize each other. This leads to a significant reduction of the intrinsic stress between films and substrates, improving on the adhesion of the films, as well as to a substantial increase of amorphous state stability of structural network.

The success of micro- and nanoelectronics device creation is largely determined by the capabilities and effectiveness of the material properties control. The generally accepted method of controlling the properties of crystalline semiconductors is the controlled addition of small amounts of donor or acceptor impurities. However, soon after the discovery of non-crystalline (amorphous) semiconductors, a weak sensitivity to the donor and acceptor impurities of these materials was established. It should be noted that alternative methods of controlling properties have been developed for a number of non-crystalline semiconductors. Such methods include chemical modification of properties for chalcogenide glassy semiconductors (CGS), structural modification of properties for amorphous carbon, hydrogenated amorphous silicon and CGS (Popov 2018). However, for other non-crystalline semiconductor materials, this question remains open to the present.

The report discusses the possibilities of controlling the properties of diamond-like silicon-carbon films by the methods of physical (structural) modification, chemical modification, and physical-chemical modification.

\section{SAMPLE PREPARATION}

Silicon-carbon films were prepared by plasma decomposition of a silicon-containing organic matter (polyphenylmethylsiloxane, PPMS, or polymethylsiloxane, PMS). During the film deposition process negative voltage was applied to the substrate holder. To obtain metal-based nanocomposites metal magnetron sputtering was conducted simultaneously with the growth of the silicon-carbon films. Schematic 
illustration of sample preparation system is shown in Fig. 1. It provides an opportunity to produce metalcontaining films with different metal contents in a single technological process. It gives the opportunity to obtain metal-containing films with different metal content in a single technological process due to fixed position of the substrate holder.

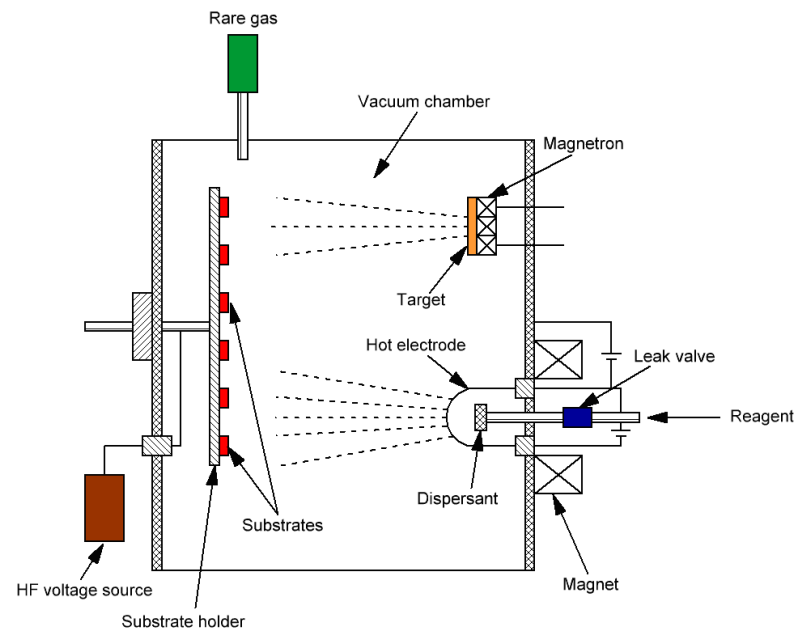

Figure 1: Schematic illustration of sample preparation system

\section{METHODS OF MODIFICATION}

\subsection{Physical modification}

The physical (structural) modification of non-crystalline material's properties allows to control properties by changing the material structure without changing its chemical composition. The physical base for the method is the fact that free energy of non-crystalline semiconductors has several minima corresponding to various metastable states of the system. Different structure of the material is obtained by varying the technological regimes and / or conditions of the material preparation. In this work, changes in the structure of amorphous diamond-like silicon-carbon films were achieved by varying the bias voltage on the substrate holder during film growth (from -100 to $-1000 \mathrm{~V}$ ) and bombarding the growing film with argon ions (the partial pressure of argon in the working chamber varied from 0 to $7 \cdot 10^{-4}$ Torr).

The magnitude of the potential on the substrate holder determines the kinetic energy (and hence the mobility) of particles falling on the substrate during the growth of the film. The bombardment of the growth surface with argon ions should affect the film structure. It is shown that the variation of the above parameters leads to a change in the morphology of the sample surface, mechanical properties and electrical conductivity of the material. An increase in the bias potential of the substrate holder leads to a decrease in the surface waviness of the samples by more than 2 times (from 0.9 to $0.4 \mathrm{~nm}$ ) (Fig. 2). The nanohardness of films increases from 10-13 GPa to 18-24 GPa (Zavedeev et al. 2017) and the electrical conductivity decreases in by at least an order of magnitude (from $10^{-12}$ to $10^{-13} \mathrm{~S} / \mathrm{cm}$ at 300 K) (Fig. 3).

Even greater changes in the properties are observed when a growing film is bombarded with argon ions: as the pressure of argon in the chamber increases from 0 to $7 \cdot 10^{-4}$ Torr (with a potential on the substrate holder of $-200 \mathrm{~V}$ ), the waviness of the film surface increases from $0.9 \mathrm{~nm}$ to $1.3 \mathrm{~nm}$ (Fig. 2), and the electrical conductivity increases by 6 orders of magnitude (from $10^{-12}$ to $10^{-6} \mathrm{~S} / \mathrm{cm}$ ) (Fig. 3).

To establish the causes of the effect of argon pressure in the chamber on the magnitude and activation energy of the electrical conductivity of silicon-carbon films, spectroscopy of deep levels in the band gap of the material was carried out using charge deep-level transient spectroscopy method (Q-DLTS) (Fig. 4).

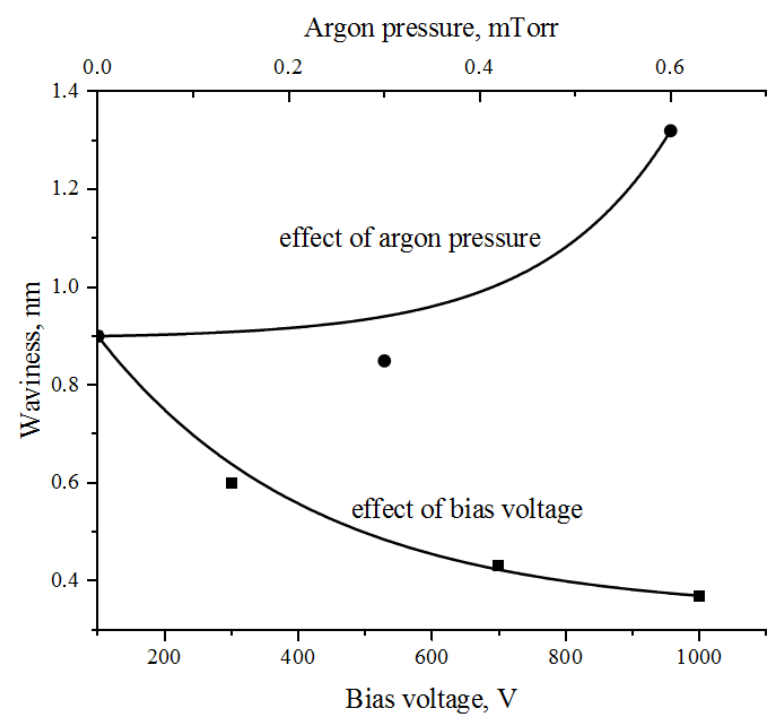

Figure 2: Dependence of the waviness of the film surface on the bias voltage applied to the substrate holder and on partial pressure of argon in the reactor

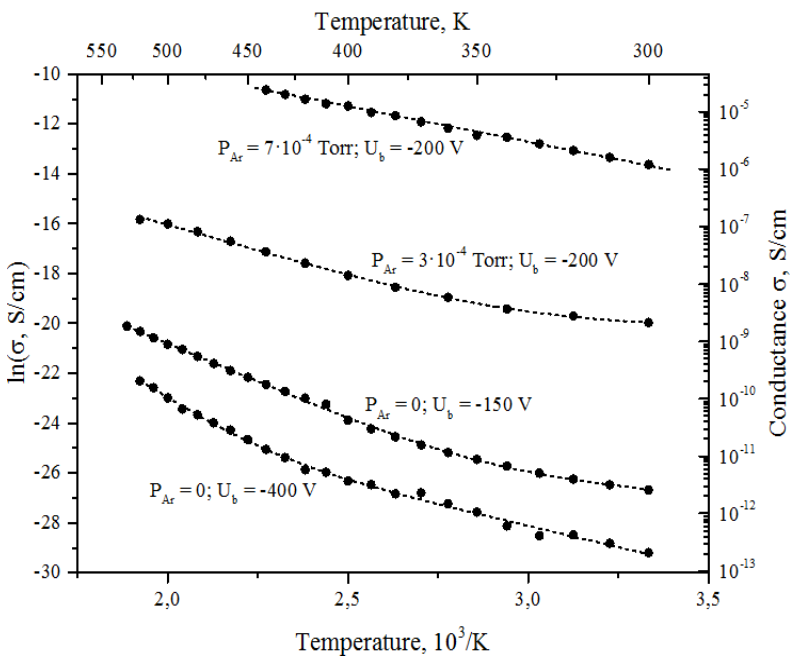

Figure 3: Temperature dependences of the conductivity of silicon-carbon films prepared at different values of bias voltage applied to the substrate holder and on partial pressure of argon in the reactor 


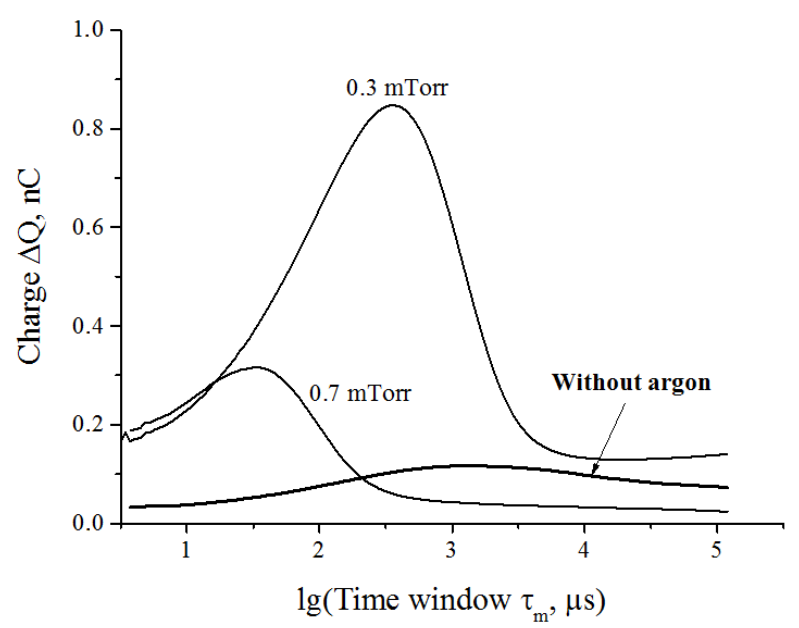

Figure 4: Q-DLTS spectra

Analysis of the Q-DLTS spectra showed that localized states in films prepared both with argon and without argon in the chamber is most likely to have the same nature and is due to the presence of dangling bonds. The concentration of localized states in the film increases with increasing argon pressure in the chamber.

Thus, the method of physical modification makes it possible to control in a significant extent the properties of diamond-like silicon-carbon films without changing their chemical composition. The total changes in electrical conductivity using the above physical modification factors are 7-8 orders of magnitude.

\subsection{Chemical modification}

The chemical modification of properties involves the introduction of additional chemical elements in the films during their preparation. In this work, metalcontaining silicon-carbon films were fabricated by plasma-chemical decomposition of a silicon-organic precursor with simultaneous magnetron sputtering of transition metals (titanium, vanadium, molybdenum, hafnium, tantalum, tungsten). The metal concentration ranged from 4 to 35 at. \%. The study of the structure of samples by high-resolution electron microscopy and X-ray photoelectron spectroscopy showed that the introduction of all metals into the silicon-carbon matrix throughout the entire concentration range leads to the formation of a two-phase system: an amorphous matrix with metal carbide nanocrystals embedded in it (Fig. 5). The size of nanocrystals was a few nanometers. No other crystalline inclusions were found in the examined samples. However, X-ray photoelectron spectroscopy spectra obtained in the region of bond energy of metal atoms showed that (at least in some cases) metaloxygen bonds are present in the samples along with metal-carbon bonds. For example, in the spectra of tungsten-containing samples there are two distinct peaks (Fig. 6), the position of which almost coincides with the energy range of chemical bonds in the tungsten oxide WO3. This indicates that, in these samples, along with the nanocrystalline carbide phase, there is an oxide phase.
However, since high resolution electron microscopy and diffraction analysis reveal only metal carbide nanocrystals in samples, it should be assumed that the oxide phase is in an amorphous state. Simulation of Xray photoelectron spectroscopy spectra showed approximately equal distribution of tungsten atoms between the carbide and oxide phases.

The carbides of transition metals formed upon the introduction of these metals into the dielectric amorphous silicon-carbon matrix are crystals with high electrical conductivity. In this regard, the use of these metals for the chemical modification of the electrophysical properties of silicon-carbon films seems to be effective. Studies of the concentration dependences of electrical conductivity showed (Fig. 7) that in all cases these dependences are of percolation type, and the value of electrical conductivity with the introduction of metal is up to 30-35 at. \% increases by 9 orders of magnitude from $10^{-6}$ to $10^{3} \mathrm{~S} / \mathrm{cm}$.

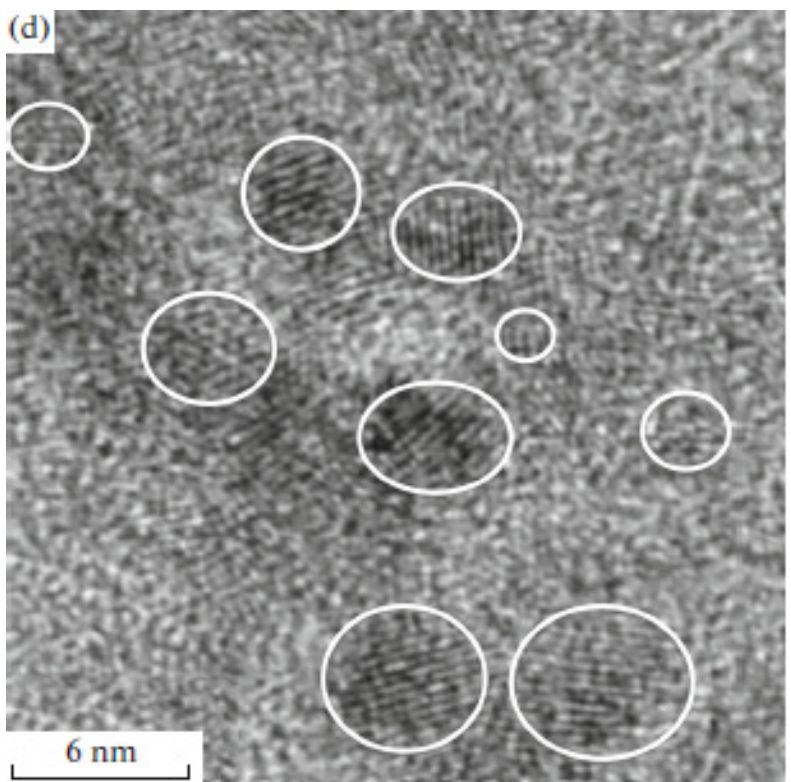

Figure 5: High resolution transition electron microscopy image of tantalum containing silicon-carbon film (Barinov, Popov et al. 2017)

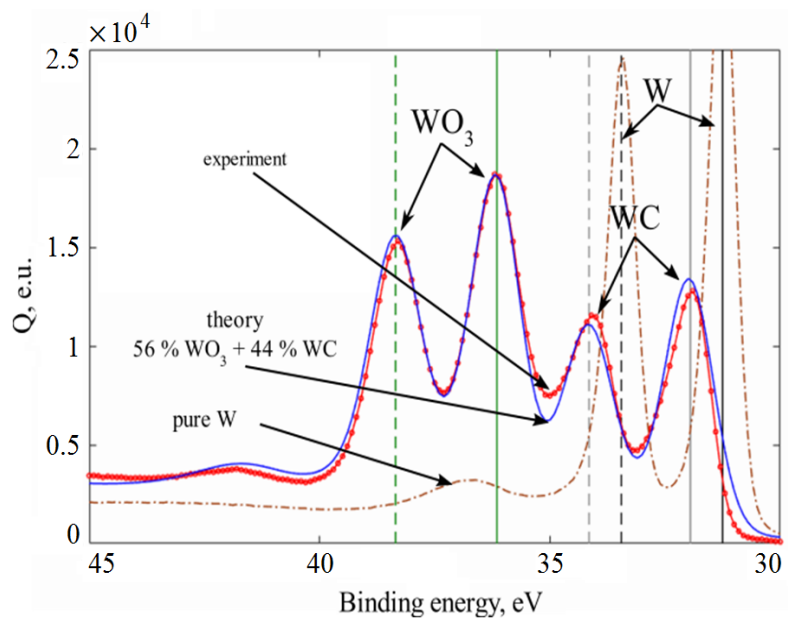

Figure 6: XPS spectra $\mathrm{W} 4 f_{5 / 2}$ and $4 f_{7 / 2}$ 


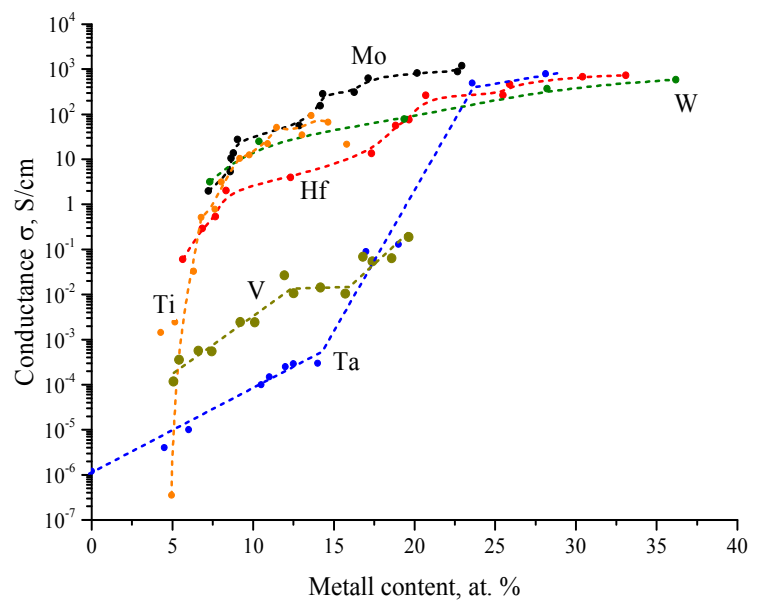

Figure 7: Dependences of silicon-carbon films conductivity on the concentration of metals

\subsection{Physical-chemical modification}

It should be noted that a number of experimental results obtained when introducing metals into silicon-carbon films are difficult to explain without taking into account changes not only in chemical composition, but also in the structure of the samples. For example, such results are dependencies of mechanical properties on metal concentration. As mentioned above, transition metals in silicon-carbon films form the nanocrystalline phase in the form of carbides. The carbon atoms for the formation of carbides are borrowed from the siliconcarbon matrix. Thus, despite the unchanged total concentration of carbon atoms, it is redistributed between the silicon-carbon amorphous matrix and the carbide nanocrystals of the introduced metal. Based on studies of the concentration dependences of the electrophysical and mechanical properties, it was shown that to correctly explain the dependencies of properties on metal concentration, it is necessary to take into account not only the presence and amount of the nanocrystalline phase, but also changes in the properties of the silicon-carbon matrix due to its structural changes as a result of carbon depletion.

Dependences of microhardness and modulus of elasticity of molybdenum- and tungsten-containing silicon-carbon nanocomposites on the metal concentration are shown in Fig. 8. An increase in the metal content leads to a redistribution of carbon in the nanocomposite: its concentration in the conducting nanocrystalline carbide phase increases, and in the amorphous silicon-carbon matrix it decreases. This leads to the appearance of two competing processes: on the one hand, an increase in the share of the carbide phase increases its contribution to the mechanical properties of the nanocomposite, and on the other hand, the depletion of the matrix with carbon causes a decrease in its contribution to the mechanical properties. These processes cause the appearance of an extremum on the concentration dependences of the mechanical properties (nanohardness and elastic modulus). However, as can be seen from Fig. 8, the minimum is observed only in the case of films with molybdenum, but it is absent in films with tungsten. To explain this fact, it should be noted that the size of nanoparticles in films with molybdenum is about $2.5 \mathrm{~nm}$, and in films with tungsten - about $1 \mathrm{~nm}$ (Barinov, Popov et al. 2017). Therefore, with an equal content of the conducting phase (MoC and $\mathrm{BC}$ ), the amount of tungsten carbide nanoparticles will be larger than that of molybdenum carbide. And this will cause a more rapid increase in the influence of the carbide phase on the mechanical properties of the material and the absence of an extremum on the concentration dependence for samples with tungsten.
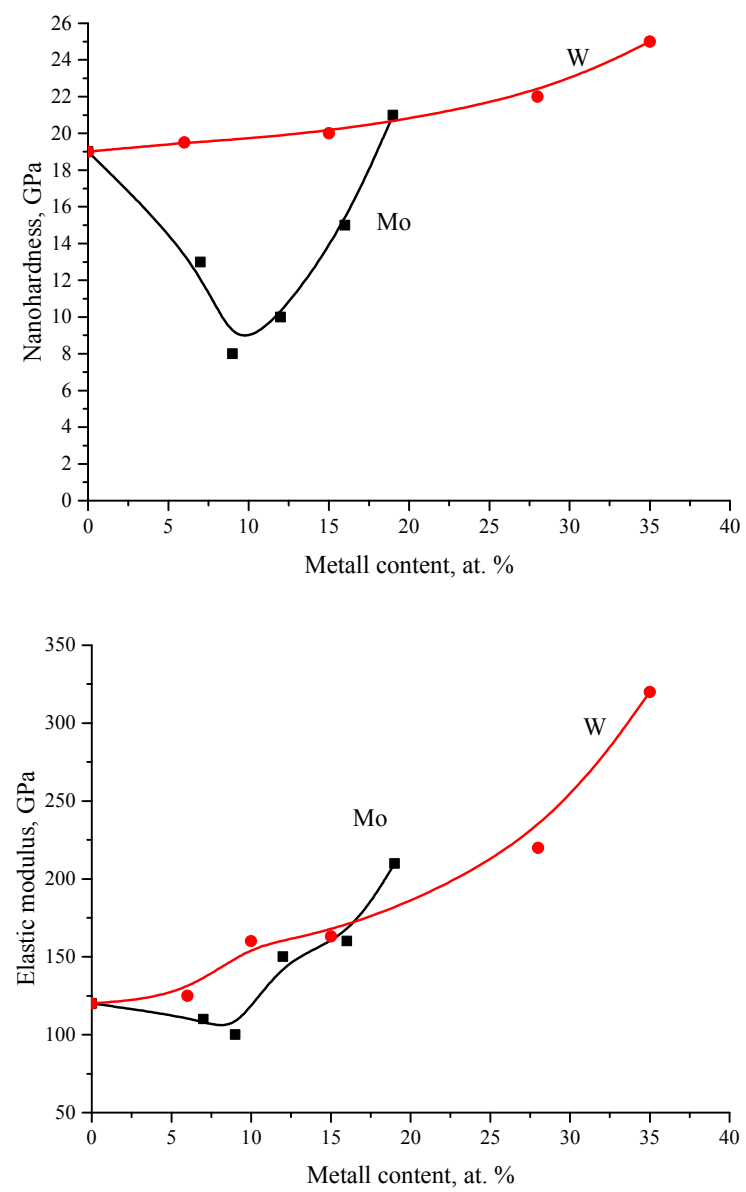

Figure 8: Mechanical properties as functions of metal concentration for the molybdenum- and tungstencontaining nanocomposites (nanohardness - upper and elastic modulus - lower)

\section{CONCLUSION}

Thus, the proposed methods provide wide possibilities for controlling the physical properties of diamond-like silicon-carbon films. For example, the range of possible changes in electrical conductivity with a combination of different control methods is 16 orders of magnitude. This opens up the possibility of widespread use of these materials in micro- and nanoelectronics.

\section{ACKNOWLEDGMENTS}

The authors are grateful to M.L. Shupegin for preparing the samples. This research was financially supported by 
the Russian Foundation for Basic Research (research project No. 19-07-00021), the Ministry of Education and Science of the Russian Federation (research project No. 2.3208.2017/4.6), and the Council on grants of the President of the Russian Federation (research project No. SP-834.2019.3).

\section{REFERENCES}

Barinov A.D., Popov A.I. and Presniakov M.Yu., 2017. Effect of nanophase concentration on the properties of metal-containing silicon-carbon nanocomposites. Inorganic Materials, 53 (7), 690696.

Mangolinia F., Krick B.A. et al., 2018. Effect of silicon and oxygen dopants on the stability of hydrogenated amorphous carbon under harsh environmental conditions. Carbon, 130, 127-136.

Popov A., 2018. Disordered semiconductors: Physics and applications. 2nd ed. Singapore: Pan Stanford Publishing.

Robertson J., 2002. Diamond-like amorphous carbon. Materials Science and Engineering, 37, 129-281.

Zavedeev E.V., Zilova O.S., Barinov A.D. et al., 2017. Femtosecond laser microstructuring of diamondlike nanocomposite films. Diamond \& Related materials, 74, 45-52.

\section{AUTHORS BIOGRAPHY}

Alexey D. Barinov was born in Moscow in 1989. After graduating from the Moscow Power Engineering Institute (Technical University) he obtained his $\mathrm{PhD}$ degree in the field of semiconductor physics in 2017. He currently works as an Assistant Professor at the same university. In addition, he works as a Researcher at the Institute of Nanotechnology of Microelectronics of the Russian Academy of Science. His research interests relate to the study of the electrophysical and morphological properties of semiconductor and dielectric thin films.

Anatoly I. Popov was born in Moscow, in 1944. After graduating from the Moscow Power Engineering Institute (Technical University) in 1968, he then obtained his $\mathrm{PhD}$ and Doctor of Science degrees in physics of semiconductors and insulators from the same university in 1973 and 1984 respectively. He is now a Professor at the National Research University "Moscow Power Engineering Institute" since 1987. He also holds position of Principle Research Scientist at the Institute of Nanotechnology of Microelectronics of the Russian Academy of Science. His scientific interests lie in exploration of the relationship between atomic structure and physical properties of disordered semiconductor materials and in development of optical storage and electrical memory devices based on these materials.

Alexander A. Makarov was born in 1993. He received the B.Sc. and the M.Sc. degrees from the National Research University "Moscow Power Engineering Institute" in 2014 and 2016, respectively, both in radio engineering. Presently, Alexander A. Makarov is a $\mathrm{PhD}$ student in the Department of Electronics and Nanoelectronics at the National Research University "Moscow Power Engineering Institute" as well as he is working as a Researcher at the International Laboratory of Statistics of Stochastic Processes and Quantitative Finance of the National Research Tomsk State University. His research interests include synthesis, analysis and simulation of the processing algorithms of stochastic processes and fields with unknown parameters. 